\title{
Calora: A Software to Simulate Calcium Diffusion
}

\author{
Gaurav Gupta (Corresponding author) \\ Department of Mathematics \\ Maulana Azad National Institute of Technology \\ Bhopal - 462051, India \\ Tel: 91-99939-03363 E-mail: gaurav_gaurav2@yahoo.co.in \\ Shivendra Tewari \\ Department of Mathematics \\ Maulana Azad National Institute of Technology \\ Bhopal - 462051, India \\ Tel: 91-94257-27127Ｅ-mail: shivendra.tewari@rediffmail.com \\ K.R. Pardasani \\ Department of Mathematics \\ Maulana Azad National Institute of Technology \\ Bhopal - 462051, India \\ Tel: 91-94253-58308Ｅ-mail: kamalrajp@rediffmail.com
}

\begin{abstract}
Calcium is a vital second messenger which regulates processes in almost all cell types like myocytes, hepatocytes, oocytes,neurons etc. Due to spatiotemporal limitation of imaging techniques, it is not possible to determine calcium concentration at macroscopic level. For this purpose mathematical modeling of calcium dynamics provides a suitable alternative. Several mathematical models governing intracellular $\mathrm{Ca}^{2+}$ dynamics have been proposed thus far. Here in this paper author has developed a software to simulate two distinct approximations, namely, Excess buffering approximation (EBA), and Rapid Buffering approximation (RBA). The software is based on oracle 10g and follows an algorithm based on an explicit Eulers method. The software contains all the important functionalities, like Multiple graphs, Spatio-temporal zoom, desired $\mathrm{Ca}^{2+}$ conc. value retrieval at specific space \& time etc., to simulate the approximations for all important biophysical constants, to have a graphical representation of a simulated model and to do comparative analysis among the mathematical models.
\end{abstract}

Keywords: $\mathrm{Ca}^{2+}$ Diffusion, Oracle, Partial differential equation

\section{Introduction}

Calcium ions $\left(\mathrm{Ca}^{2+}\right)$ impact nearly every aspect of cellular life as they are fundamental important component of cellular signal transduction. It helps in regulating cell growth and differentiation. (Lodish, Berk, Zipursky, Matsudaira, Baltimore, Darnell, 2000). The divalent cation calcium $\left(\mathrm{Ca}^{2+}\right)$ is also used by cells as a second messenger to control many cellular processes including muscle contraction, secretion, metabolism, neuronal excitability, cell proliferation, and cell death (Simpson, Challiss \& Nahorski, 1995).

Neuronal activity can lead to marked increases in the concentration of cytosolic calcium. Calcium binds to calmodulin and stimulates the activity of a variety of enzymes, including calcium-calmodulin kinases and calcium-sensitive adenylate cyclases. These enzymes transduce the calcium signal and effect short-term biological responses, such as the modification of synaptic proteins and long-lasting neuronal responses that require changes in gene expression. The calcium levels outside cells are 10000 times higher than free intracellular $\mathrm{Ca}^{2+}$. However, free $\left[\mathrm{Ca}^{2+}\right]_{\mathrm{i}}$ is the physiologically active form of calcium (Rasmussen, 1988). The level of free intracellular calcium $\left(\left[\mathrm{Ca}^{2+}\right]_{\mathrm{i}}\right)$ is regulated and maintained as low as $(\sim 100 \mathrm{nM})$ through the action of a number of binding proteins and ion exchange mechanisms. 
Each cell has a unique set of $\mathrm{Ca}^{2+}$ signals to control its function. $\mathrm{Ca}^{2+}$ signal transduction is based on rises in free cytosolic $\mathrm{Ca}^{2+}$ concentration. $\mathrm{Ca}^{2+}$ can flow from the extracellular space or be released from intracellular stores. The endoplasmic reticulum (ER) is a major site for sequestered $\mathrm{Ca}^{2+}$ ions.

Calcium concentrations are strongly buffered in living cells. Buffer site concentrations have been estimated to be in the range of 100-300, $\mu \mathrm{M}$ in the cytoplasm and significantly higher in the endoplasmic reticulum (ER) (Allbritton, Meyer \& Stryer, 1992; Michalak, Milner, Burns \& Opas, 1992). Certain proteins of the cytoplasm and organelles act as buffers by binding $\mathrm{Ca}^{2+}$. Which binds most of the $\mathrm{Ca}^{2+}$ in a cell (up to 99\%). Depending on their diffusion characteristics, buffers are considered as mobile or immobile. Moreover, buffers can control intracellular Calcium dynamics by tuning spatial coupling of channel clusters. That determines the average period of oscillations due to wave nucleation (Falcke, 2003; Lukyanenko \& Gyorke, 1999).

Two theories have been used to simplify the system of reaction-diffusion equations governing calcium diffusion. One is excess buffer approximation (EBA) which assumes that mobile buffer is present in excess and cannot be saturated. The other is rapid buffer approximation (RBA), which assumes that calcium binding to buffer is rapid compared to calcium diffusion rate. In the present work an attempt has been made to develop software which simulates $\mathrm{Ca}^{2+}$ diffusion under both type of approximation.

EBA is appropriate when the saturability of mobile buffer is negligible and when there is significant saturability of mobile buffer and buffer kinetics are fast relative to $\mathrm{Ca}^{2+}$ diffusion then RBA is appropriate (Tewari,Tewari \& Pardasani, 2009) . In, our software, we have a single interface via which users can switch to either of the approximation. Further, the software is developed using Oracle $10 \mathrm{~g}$ to provide handling of large databases generated by model simulation.

\section{Mathematical Formulation}

Author has assumed that only $\mathrm{Ca}^{2+}$ ions and buffers are present inside the cytosol. Assuming the following reaction equation between $\mathrm{Ca}^{2+}$ and buffers,

$$
\left[\mathrm{Ca}^{2+}\right]+\left[B_{j}\right] \text { 日照到 }\left[\mathrm{CaB}_{j}\right]
$$

Where $\left[\mathrm{B}_{\mathrm{j}}\right]$ and $\left[\mathrm{CaB}_{\mathrm{j}}\right]$ are free and bound buffer respectively and ' $\mathrm{j}$ ' is an index over buffer species. If we further assume that the cytosolic medium is homogenous and is isotropic then, Calcium dynamics in presence of a point source can be framed in the form of following equations (Neher 1986; Smith 1996; Smith, Dai, Miura \& Sherman 2000):

$$
\begin{aligned}
& \frac{\partial\left[C a^{2+}\right]}{\partial t}=D_{C a} \nabla^{2}\left[C a^{2+}\right]+\sum_{j} R_{j}+\delta \sigma(r) \\
& \frac{\partial\left[B_{j}\right]}{\partial t}=D_{B_{j}} \nabla^{2} B_{j}+R_{j} \\
& \frac{\partial\left[C a B_{j}\right]}{\partial t}=D_{C a B_{j}} \nabla^{2}\left[C a B_{j}\right]-R_{j}
\end{aligned}
$$

Where, the Laplacian operator written in spherical symmetry,

$$
\begin{aligned}
& \nabla^{2}=\frac{\partial^{2}}{\partial r^{2}}+\frac{2}{r} \frac{\partial}{\partial r} \\
& R_{j}=-k_{j}^{+}\left[B_{j}\right]\left[C a^{2+}\right]+k_{j}^{-}\left[C a B_{j}\right]
\end{aligned}
$$

$\mathrm{D}_{\mathrm{Ca}}, \mathrm{D}_{\mathrm{Bj}}, \mathrm{D}_{\mathrm{CaBj}}$ are diffusion coefficient of free calcium, free buffer, and $\mathrm{Ca}^{2+}$ bound buffer, respectively; $\mathrm{k}_{\mathrm{j}}^{+}$and $\mathrm{k}_{\mathrm{j}}^{-}$are association and dissociation rate constants for buffer ' $\mathrm{j}$ ', respectively. For stationary, immobile buffers or fixed buffers $\mathrm{D}_{\mathrm{Bj}}=\mathrm{D}_{\mathrm{CaBj}}=0$.

For boundary conditions, we assume a point source of $\mathrm{Ca}^{2+}$ at origin and a fixed background $\mathrm{Ca}^{2+}$ concentration. There is no source for buffer, and the buffer is assumed to be in equilibrium with $\mathrm{Ca}^{2+}$ far from the source. The corresponding equation is (Smith et al., 2000)

$$
\begin{array}{ll}
\lim _{r \rightarrow 0}\left\{-2 \pi r^{2} D_{C a} \frac{d\left[\mathrm{Ca}^{2+}\right]}{d r}\right\}=\sigma, & \lim _{r \rightarrow \infty}\left[\mathrm{Ca}^{2+}\right]=\left[\mathrm{Ca}^{2+}\right]_{\infty}, \\
\lim _{r \rightarrow 0}\left\{-2 \pi r^{2} D_{B_{j}} \frac{d\left[B_{j}\right]}{d r}\right\}=0, & \lim _{r \rightarrow \infty}\left[B_{j}\right]=\left[B_{j}\right]_{\infty} \\
\lim _{r \rightarrow 0}\left\{-2 \pi r^{2} D_{C a B_{j}} \frac{d\left[C a B_{j}\right]}{d r}\right\}=0, & \lim _{r \rightarrow \infty}\left[\mathrm{CaB} B_{j}\right]=\left[\mathrm{CaB} B_{j}\right]_{\infty},
\end{array}
$$




$$
\left.\left[B_{j}\right]_{\infty}=\frac{K_{j}\left[B_{j}\right]_{T}}{K_{j}+\left[\mathrm{Ca}^{2+}\right]_{\infty}}, \quad[\mathrm{CaB}]_{j}\right]_{\infty}=\frac{\left[\mathrm{Ca}^{2+}\right]_{\infty}\left[B_{j}\right]_{T}}{K_{j}+\left[\mathrm{Ca}^{2+}\right]_{\infty}}
$$

$K_{j}=k_{j}{ }^{-} / k_{j}{ }^{+}$is dissociation constant for buffer $\mathrm{j}$, and $\left[B_{j}\right]_{T}=\left[B_{j}\right]+\left[C a B_{j}\right]$ total concentration profile for each buffer.

If we assume that the diffusion constant of each mobile buffer is not affected by the binding of $\mathrm{Ca}^{2+}$ (that is, $\mathrm{D}_{\mathrm{Bj}}=\mathrm{D}_{\mathrm{CaBj}}$ ), and also assume that, $\left[\mathrm{B}_{\mathrm{j}}\right]_{\mathrm{T}}$ is initially uniform, then $\left[\mathrm{B}_{\mathrm{j}}\right]_{\mathrm{T}}$ will remain uniform for all time. Thus we can write (Smith 1996; Smith et al., 2000)

$$
R_{j}=-k_{j}^{+}\left[C a^{2+}\right]\left[B_{j}\right]+k_{j}^{-}\left(\left[B_{j}\right]_{T}-\left[B_{j}\right]\right)
$$

Finally, we restrict consideration to unsteady state i.e. to the domain of high $\mathrm{Ca}^{2+}$, done in microsecond near sources. Note that the fixed buffer, while indispensable for the time dependent PDE solution, have no influence on steady states because $R_{j}=0$ if $D_{B j}=D_{C a B j}=0$. For ease, we assume a single mobile buffer species, [B], resulting in following boundary-value problem (Wagner \& Keizer, 1994; Smith, Wagner, \& Keizer, 1996)

$$
\begin{gathered}
\frac{\partial\left[\mathrm{Ca}^{2+}\right]}{\partial t}=D_{C a} \nabla^{2}\left[\mathrm{Ca}^{2+}\right]-k^{+}[B]\left[\mathrm{Ca}^{2+}\right]+k^{-}\left([B]_{T}-[B]\right), \\
\frac{\partial[B]}{\partial t}=D_{B} \nabla^{2}[B]-k^{+}[B]\left[C a^{2+}\right]+k^{-}\left([B]_{T}-[B]\right),
\end{gathered}
$$

With these boundaries condition (Smith et al., 2000)

$$
\begin{array}{ll}
\lim _{r \rightarrow 0}\left\{-2 \pi r^{2} D_{C a} \frac{d[C a]^{2+}}{d r}\right\}=\sigma, & \lim _{r \rightarrow \infty}\left[C a^{2+}\right]=\left[C a^{2+}\right]_{\infty}, \\
\lim _{r \rightarrow 0}\left\{-2 \pi r^{2} D_{B} \frac{d[B]}{d r}\right\}=0, & \lim _{r \rightarrow \infty}[B]=[B]_{\infty}=\frac{K[B]_{T}}{K+\left[C a^{2+}\right]_{\infty}} .
\end{array}
$$

Basically there are two approximations which have been proposed in order to simplify equation (1-4),

\subsection{The Excess Buffer Approximation (EBA):}

In seminal work Neher (1986) made the critical observation that if buffer is present in excess then, free mobile buffer profile is not perturbed by presence of the source. Under this assumption, one can make the approximation that $[\mathrm{B}] \approx[\mathrm{B}]_{\infty}$

$$
\frac{\partial\left[C a^{2+}\right]}{\partial t}=D_{C a} \nabla^{2}\left[C a^{2+}\right]-k^{+}[B]_{\infty}\left[C a^{2+}\right]+k^{-}\left([B]_{T}-[B]_{\infty}\right) .
$$

As $t \rightarrow \infty$ one can write

$$
\begin{gathered}
k^{-}\left([B]_{T}-[B]_{\infty}\right)=k^{+}[B]_{\infty}\left[C a^{2+}\right]_{\infty}, \\
\frac{\partial\left[C a^{2+}\right]}{d t}=D_{C a} \nabla^{2}\left[C a^{2+}\right]-k^{+}[B]_{\infty}\left(\left[C a^{2+}\right]-\left[C a^{2+}\right]_{\infty}\right)
\end{gathered}
$$

This linear equation for $\left[\mathrm{Ca}^{2+}\right]$ at steady state can be solved easily and applying the boundary conditions would yield (Smith 1996),

$$
\left[C a^{2+}\right]=\frac{\sigma}{2 \pi D_{C a} r} e^{-r / \lambda}+\left[C a^{2+}\right]_{\infty}
$$

Where $\lambda$ is the characteristic length constant for the mobile $\mathrm{Ca}^{2+}$ buffer given by $\lambda=\sqrt{D_{C a} / k^{+}[B]_{\infty}}$. This approximation has been shown to be valid when mobile buffer is in high concentration and/or when the source amplitude is small, that is, $\lim _{r \rightarrow 0}[B] \approx[B]_{\infty}$

Initial condition is

And boundary condition

$$
\left[\mathrm{Ca}^{2+}\right]_{t=0}=0.1 \mu \mathrm{M}
$$




$$
\begin{aligned}
& \lim _{r \rightarrow 0}\left(-2 \pi D_{C a} r^{2} \frac{d\left[\mathrm{Ca}^{2+}\right]}{d r}\right)=\sigma_{C a} \\
& \lim _{r \rightarrow \infty}\left[\mathrm{Ca}^{2+}\right]=0.1 \mu \mathrm{M}
\end{aligned}
$$

\subsection{Rapid Buffer Approximation (RBA):}

In Rapid Buffering approximation, we assume that the buffering time scales are rapid, reaching equilibrium at each point in space before appreciable diffusion occurs. This means that the buffering is a singular perturbation (Murray, 2001), which we handle by deriving an equation for the local, total concentration of $\mathrm{Ca}^{2+},\left[\mathrm{Ca}^{2+}\right]_{\mathrm{T}}$ :

$$
\left[\mathrm{Ca}^{2+}\right]_{T}=\left[\mathrm{Ca}^{2+}\right]+[\mathrm{CaB}]+\left[\mathrm{CaB}_{m}\right]
$$

We find that

$$
\frac{\partial\left[\mathrm{Ca}^{2+}\right]_{T}}{\partial t}=D_{C a} \nabla^{2}\left[\mathrm{Ca}^{2+}\right]+D_{C a B_{m}} \nabla^{2}\left[\mathrm{CaB}_{m}\right]
$$

This equation does not involve the rapid buffering time scale and, therefore, involves no singular perturbation. It is possible to eliminate $\left[\mathrm{CaB}_{\mathrm{m}}\right]$ from this equation using the assumption of rapid equilibrium (Wagner and Keizer 1994),

$$
\left[\mathrm{CaB}_{i}\right]=\frac{\left[\mathrm{Ca}^{2+}\right]\left[\mathrm{B}_{i}\right]_{T}}{\mathrm{~K}_{\mathrm{i}}+\left[\mathrm{Ca}^{2+}\right]}
$$

Where $\mathrm{K}_{\mathrm{i}}$ is the dissociation constant and $\left[\mathrm{B}_{\mathrm{j}}\right]_{\mathrm{T}}$ is the total concentration of stationary or mobile buffer binding sites. When combined allows $\left[\mathrm{Ca}^{2+}\right]_{\mathrm{T}}$ to be written in terms of $\left[\mathrm{Ca}^{2+}\right]$,

Using equation 21 , one can write,

$$
\left[\mathrm{Ca}^{2+}\right]_{\mathrm{T}}=\left[\mathrm{Ca}^{2+}\right]\left(1+\frac{\left[B_{s}\right]_{T}}{\left(K_{s}+\left[\mathrm{Ca}^{2+}\right]\right)}+\frac{\left[B_{m}\right]_{T}}{\left(K_{m}+\left[\mathrm{Ca}^{2+}\right]\right)}\right)
$$

The following transport equation for free $\mathrm{Ca}^{2+}$ :

$$
\begin{gathered}
\frac{\partial\left[\mathrm{Ca}^{2+}\right]}{\partial t}=\beta\left(D_{C a}+\gamma_{m} D_{C a B_{m}}\right) \nabla^{2}\left[\mathrm{Ca}^{2+}\right]-\frac{2 \beta \gamma_{m} D_{C a B_{m}}}{K_{m}+\left[\mathrm{Ca}^{2+}\right]}\left(\nabla\left[\mathrm{Ca}^{2+}\right]\right)^{2}, \\
\gamma_{i}=\frac{k_{i}[B]_{T}}{\left(k_{i}+\left[\mathrm{Ca} a^{2+}\right]\right)^{2}}, \beta=\left(1+\gamma_{m}+\gamma_{s}\right)^{-1}
\end{gathered}
$$

Where, $i=m$ or s. Assuming that $D_{\mathrm{CaBm}}$ and $\mathrm{D}_{\mathrm{Bm}}$ is approximately equal so we can rewrite this equation in this form along with initial boundary condition (Wagner and Keizer 1994; Smith et al., 1996)

Initial Condition,

$$
\frac{\partial\left[\mathrm{Ca}^{2+}\right]}{\partial t}=\beta\left(D_{C a}+\gamma_{m} D_{B m}\right) \nabla^{2}\left[\mathrm{Ca}^{2+}\right]-\frac{2 \beta \gamma_{m} D_{B m}}{K_{m}+\left[\mathrm{Ca}^{2+}\right]}\left(\nabla\left[\mathrm{Ca}^{2+}\right]\right)^{2},
$$

$$
\left[\mathrm{Ca}^{2+}\right]_{t=0}=0.1 \mu \mathrm{M},
$$

And boundary condition near the channel is given (Wagner and Keizer, 1994)

$$
\lim _{r \rightarrow 0}\left\{-2 \pi r^{2} \beta\left[D_{C a}+\gamma_{m} D_{B m}\right] \frac{\partial\left[C a^{2+}\right]}{\partial r}\right\}=\beta \sigma,
$$

In the next section we discuss about the importance of the software and the results obtain by the software.

\section{CalOra: The Software}

It is Oracle $10 \mathrm{~g}$ based software in which there is a single interface for both simulations i.e. rapid buffer approximation and excess buffer approximation. The user can switch in between any of the simulations at any time. To start CalOra interface, there is a need for server OC4J (Oracle container for J2EE) instance which has to be started before its interface. The OC4J standalone distribution includes an HTTP(S) server, the required J2EE services, and Web Services capabilities all of which are executed from one Java process. This server comes with the Oracle $10 \mathrm{~g}$ setup and is installed along with it. CalOra gives the result in large precision form (the number values after the decimal place) and 
this precision number can be changed according to the computational power one needs to spend.

CalOra runs on web browser and has to be configured to run in your machine. Data generated from CalOra can be saved in oracle database for future reference. Saved simulated data can be used to perform comparative studies. Figure-1 shows the main page of CalOra where user enters its choice to proceed with any of the simulations according to his/her need. It is basically software for simulating calcium diffusion obeying excess buffering and Rapid buffering approximations. Help is given in the upper right corner of CalOra where beginners may get help about EBA, RBA module and its limitations as shown in figure 2, so that the user can get familiar with the functioning of our software.

Now, if user selects EBA or RBA, he will move on to the respective module where he can enter different parametric values according to his/her need, but if user does not want to enter any values then the user can proceed with the simulation using the default values stored in the Oracle database, so that naïve user can also perform the simulation without hovering about the parametric values to choose. The user need not to enter all the parametric values and hence can change only few values to get complete results along with graphical representation of their simulation. Input page of EBA and RBA module is given in figure 3 and 4.

In EBA section the user can perform two simulation in one go. How? He can choose two different buffer association rates for two simulations and click the calculate button, to have two different tables corresponding to the two buffer association rates. User can see the simulated calcium concentration values with the help show values buttons adjacent to the table containing $\left[\mathrm{Ca}^{2+}\right]$ values. The user can see the $\left[\mathrm{Ca}^{2+}\right]$ values for both the buffer with respect to space and time and can be return back to the input page, using back button, where he/she can again make changes to the parametric values. Further the user can see the results in graphical form with the help of Graph button.

Figure 3 shows the GUI for EBA module. The values shown in the text box along with the text labels are the default parametric values stored in Oracle database. There are there buttons shown namely Calculate, $\left[\mathrm{Ca}^{2+}\right]$ Values and Graphs. Calculate button can be used, once the user has decided to calculate calcium concentration with his/her own parametric values. These values can be seen with the help of second button $\left[\mathrm{Ca}^{2+}\right]$ values. These values are shown in a table, where the rows corresponds the space node and the column correspond the time node. From the table, if user wants to know the particular value of calcium concentration on specific space and time node, he/she can use enter query and execute query buttons to see the desired value as shown in figure 5. Graph button takes the user to the graphical representation page where the user can see the simple and logarithmic graphical results of his/her simulation.

Now if we talk about RBA, we can perform comparative study between three different types of buffers namely stationary, endogenous mobile and exogenous as shown in the figure 4.We have taken only one buffer specie per simulation assuming that the other two species are absent. To perform a comparative analysis, the concentration of each single buffer species is taken to be $50 \mu \mathrm{M}$. Further the user can enter his/her choice of buffer concentration and buffer dissociation constant. In this module, we have created three tables to store values of three different simulations. In this page, we have created some more buttons like copyl and copy 2. These two buttons can be used to copy the data in Oracle database for future reference. One another extra button is there i.e. delete all data which is used to delete all data from all tables. This functionality will be used when the user is performing comparative study. $\left[\mathrm{Ca}^{++}\right]$Values button has same predefined functionality elaborated in EBA section.

In both of the modules EBA and RBA, there is a common button "Graph". This button is used to show simple linear graph and logarithmic graph. The purpose of having logarithmic graph along with the simple graph is to differentiate between the curves under undistinguishable situations. For this purpose, we have used logarithmic scale on Y axis i.e. Calcium concentration axis which will be shown in the result and discussion section. Further the user can enter any desired value of time to have its corresponding calcium diffusion path. As usual, when the user has finished playing with the software, he can use Quit button to exit from the module.

\section{Results and Discussion}

For RBA (Rapid Buffer Approximation) and EBA (Excess Buffer Approximation), the numerical values for various parameters used are given in Table 1 and Table 2. With the help of CalOra calcium concentration profiles have been plotted with respect to space which is shown in figure 6 and figure 7.

In figure 6, there are two curves shown in the figure blue (E) represents EGTA and light blue (B) represents BAPTA. In this graph, cytosolic $\left[\mathrm{Ca}^{2+}\right]$ profile is plotted for two exogenous buffers; both have same affinity for $\left[\mathrm{Ca}^{2+}\right]$ but different binding rates. 1) EGTA (Ethylene Glycol-bis(beta-aminoethyl-ether)- $N, N, N^{\prime}, N^{\prime}$-Tetra Acetate) a very slow buffer, 2) BAPTA (1,2-bis(o-minophenoxy)ethane- $N, N, N^{\prime}, N^{\prime}$-tetra acetic acid) a very fast buffer. Exogenous buffers are used to delay or accelerate the time required to achieve steady state condition. Same thing is also evident from the figure, the results are shown in two scales as to show the difference in between the chelators binding rates and the impact they have on the spatial aspect of $\left[\mathrm{Ca}^{2+}\right]$ attaining its resting concentration. It is apparent from the $\log$ plot that $\left[\mathrm{Ca}^{2+}\right]$ achieves its resting concentration profile close to the channel $1.001 \mu \mathrm{m}$ while for EGTA it relaxes around $10.01 \mu \mathrm{m}$.

In figure 7, cytosolic calcium profile for three different buffer species, namely stationary, endogenous mobile and 
exogenous buffers, under rapid buffering approximation is shown. There are three curves shown in the figure blue (T) represents Troponin-C,light green (E) represents EGTA and light blue (C) represents Calmodulin. The study was done; assuming that only single buffer specie is present at a time i.e. assuming that data to specie are absent.The curve for Troponin- $\mathrm{C}$ is closest to the $\mathrm{y}$ axis since it is a stationary buffer and its dissociation rate is on the higher side i.e. the rate of calcium dissociation is higher than its binding rate. While the other two curves for EGTA (exogenous) and Calmodulin (endogenous) are in a different league as both of them are mobile and hence affect the path of calcium diffusion as shown in figure 7. The curve for EGTA is the second closest to y axis while the curve for Calmodulin is farthest. It is because of the fact that, the dissociation constant for Calmodulin is greater than that for EGTA. Higher dissociation constant implies that there is more free calcium inside the cytosol and hence the curve for calmoduin is farthest from $y$ axis. But one can argue the fact stated above as the dissociation constant for the Troponin-C is greatest but its curve is steepest. This happens because Troponin- $\mathrm{C}$ is a stationary buffer and hence is assumed to have zero diffusion coefficients contributing nothing to calcium diffusion but affecting the time required to achieve steady state nonetheless.

\section{Future Scope}

Similar software for $\mathrm{Ca}^{2+}$ diffusion does exist and one of them is CalC - The Calcium Calculator initiated by Matveev (2002). This project is a large scale project which is under operation since 2002 where as our software is still a stub and is under development process. In future authors would like to extend their work to the extent of this well known work of $\mathrm{CalC}$ - The Calcium Calculator. So that present work could prove beneficial in diagnosis of neurodegenerative disorders, such as Alzheimer's disease (AD), Parkinson's disease (PD), amyotrophic lateral sclerosis (ALS), Huntington's disease (HD) and spinocerebellar ataxias (SCAs), present an enormous medical, social, financial and scientific problem. Recent evidence indicates that neuronal calcium $\left(\mathrm{Ca}^{2+}\right)$ signaling is abnormal in many of these disorders. Similar, but less severe, changes in neuronal $\mathrm{Ca}^{2+}$ signaling occur as a result of the normal aging process. The role of aberrant neuronal $\mathrm{Ca}^{2+}$ signaling in the pathogenesis of neurodegenerative disorders is key research area now these days (Bezprozvanny, 2009). With the help of software development we can contribute in this field. So CalOra can be extended up to the level that it would be able in disease diagnosis by simulating complete calcium dynamics of a particular person.

\section{References}

Allbritton N., Meyer T., and Stryer L. (1992). Range of messenger action of calcium ion and inositol 1, 4, 5 trisphosphate. Science. 258, 1812-1815.

Bezprozvanny I. (2009). Calcium signaling and neurodegenerative diseases. Trends in Molecular Medicine, v. 15, pp 89-100.

Falcke M. (2003). On the role of stochastic channel behavior in intracellular $\mathrm{Ca}^{2+}$ dynamics, Biophysical Journal, 84, $42-56$.

Lodish H.,Berk A.,Zipursky S.L.,Matsudaira P.,Baltimore D.,Darnell J.E. (2000). Molecular Cell Biology (4 ${ }^{\text {th }}$ edition), New York, WH Freeman \& Company.

Lukyanenko V., Györke S. (1999). $\mathrm{Ca}^{2+}$ sparks and $\mathrm{Ca}^{2+}$ waves in saponin-permeabilized rat ventricular myocytes, Journal of Physiology 521, 575-585.

Michalak M.,Milner R.,Burns K., and Opas M. (1992). Calreticulin, Journal of Biochemistry, 285, 681-692.

Matveev V. (2002). CalC Calcium Calculator [Online] Available: http://web.njit.edu/ matveev/calc.html

Murray J.D. (2001). Mathematical Biology ( $3^{\text {rd }}$ edition), New York: Springer-Verlag.

Neher E. (1986). Concentration profiles of intracellular $\mathrm{Ca}^{2+}$ in the presence of diffusible chelators. Experimental Brain Research Series, 14, 80-96.

Rasmussen. (1988). JE: Calcium and the skin. Archive of Dermatol. 124: 443-444.

Simpson P.B., Challiss R.A.J. and Nahorski S.R. (1995). Neuronal $\mathrm{Ca}^{2+}$ stores: activation and function. Trends Neuroscience, 18, 299-306.

Smith G.D. (1996). Analytical Steady-State Solution to the rapid buffering approximation near an open $\mathrm{Ca}^{2+}$ channel, Biophysical Journal, 71, 3064- 3072.

Smith G.D., Wagner J., and Keizer J. (1996). Validity of the rapid buffering approximation near a point source of calcium ions, Biophysical Journal, 70, 2527-2539.

Smith G.D., Dai L., Miura R. M., and Sherman A. (2000). Asymptotic Analysis of buffered $\mathrm{Ca}^{2+}$ diffusion near a point source. SIAM Journal of Applied of Mathematics, 61 1816-1838

Tewari V., Tewari S., and Pardasani K.R. (2009). A Model to Study the Effect of Excess buffers and $\mathrm{Na}+$ ions on $\mathrm{Ca}^{2+}$ 
diffusion in Neuron cell. International Journal of Biological and Medical Sciences, 5, 22-27.

Wagner J. and Keizer J. (1994). Effects of rapid buffers on $\mathrm{Ca}^{21}$ diffusion and $\mathrm{Ca}^{21}$ oscillations. Biophysical Journal, 67, $447-456$.

Table 1. RBA Approximation: Parameters, Values used

\begin{tabular}{|c|c|c|}
\hline Parameter & Values & Unit \\
\hline Space Initialization & 0.001 & Micro meter \\
\hline Space Termination & 1 & Micro meter \\
\hline Space step & 100 & \\
\hline No. of time Step & 10000 & \\
\hline Initial time & 0 & $\mathrm{Sec}$ \\
\hline Final time & 0.1 & $\mathrm{Sec}$ \\
\hline Initial Condition & 0.0000001 & Molar \\
\hline Mobile Buffer Association Rate & 500 & Micro molar ${ }^{-1} \sec ^{-1}$ \\
\hline Mobile Buffer Dissociation Rate & 470 & $\mathrm{Sec}^{-1}$ \\
\hline Exogenous Buffer Association Rate & 1.5 & Micro molar ${ }^{-1} \sec ^{-1}$ \\
\hline Exogenous Buffer Dissociation Rate & 0.3 & $\mathrm{Sec}^{-1}$ \\
\hline Stationary Buffer Association Rate & 90 & Micro molar ${ }^{-1} \sec ^{-1}$ \\
\hline Stationary Buffer Dissociation Rate & 300 & $\mathrm{Sec}^{-1}$ \\
\hline Diffusion Coefficient Of Calcium & 250 & Micro meter $^{2} \sec ^{-1}$ \\
\hline Diffusion Coefficient Of Mobile & 32 & Micro meter sec $^{-1}$ \\
\hline Diffusion Coefficient Of Exogenous & 113 & Micro meter $^{2} \sec ^{-1}$ \\
\hline Source & 40 & Pico ampere \\
\hline
\end{tabular}

Table 2. EBA Approximation: Parameters, Values used

\begin{tabular}{|c|c|c|}
\hline Parameter & Values & Unit \\
\hline Initial time & 0 & $\mathrm{Sec}$ \\
\hline Final time & 100 & $\mathrm{Sec}$ \\
\hline Space Bounds & {$[0.001,1]$} & Micro meter \\
\hline Time Step Length & .01 & $\mathrm{Sec}$ \\
\hline No. of time step & 10000 & \\
\hline No. of space step & 40 & \\
\hline Space Step Length & .25 & Micro meter \\
\hline Buffer Association Rate & .0015 & Micro molar ${ }^{-1} \mathrm{sec}^{-1}$ \\
\hline Buffer Concentration & 50 & Micro molar \\
\hline Diffusion Coefficient & .25 & Micro meter $^{2} \sec ^{-1}$ \\
\hline Initial Condition & .1 & Micro molar \\
\hline Source & 1 & Pico ampere \\
\hline
\end{tabular}




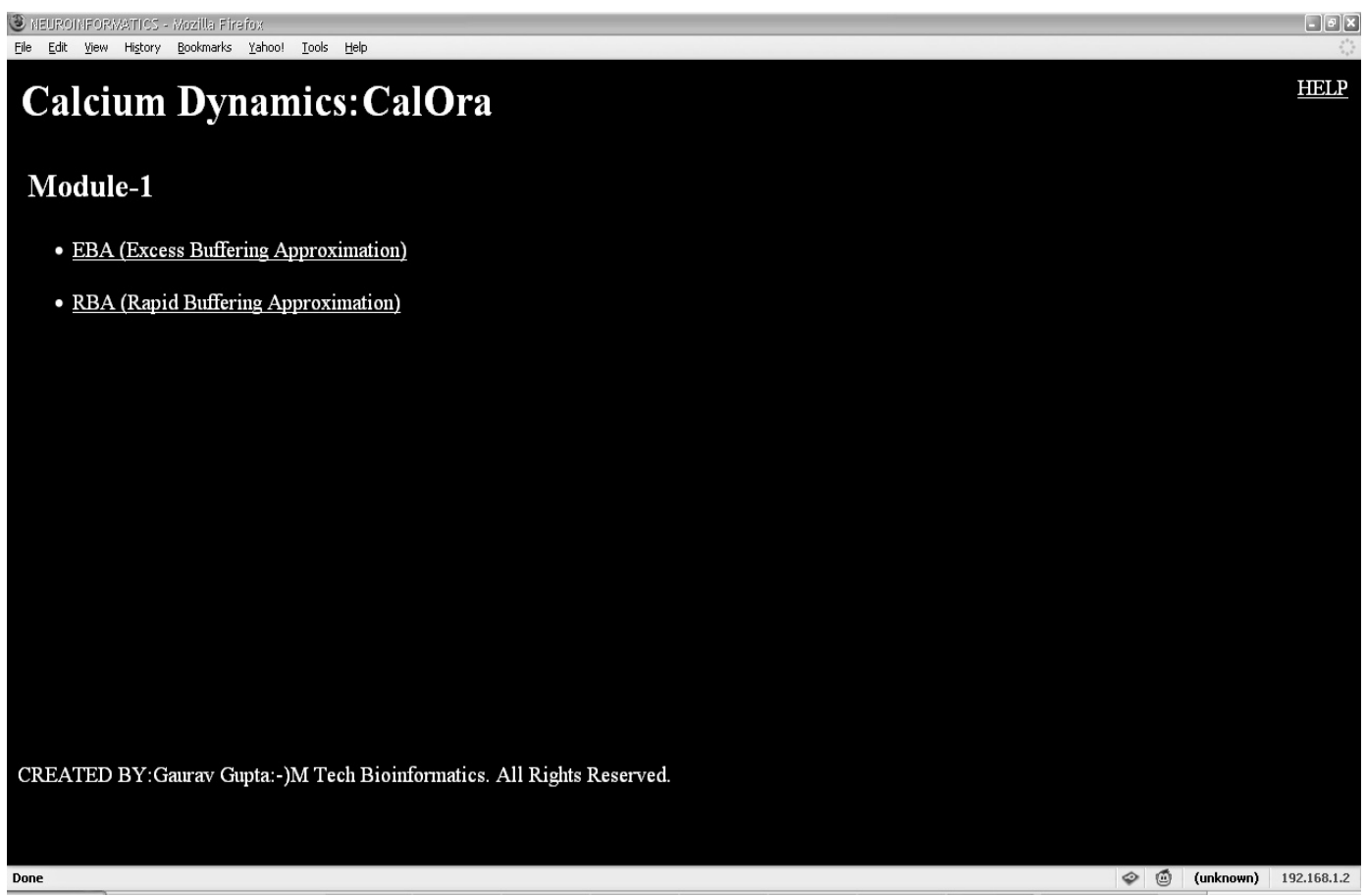

Figure 1. Webpage showing main Page of CalOra where user can switch either of simulation

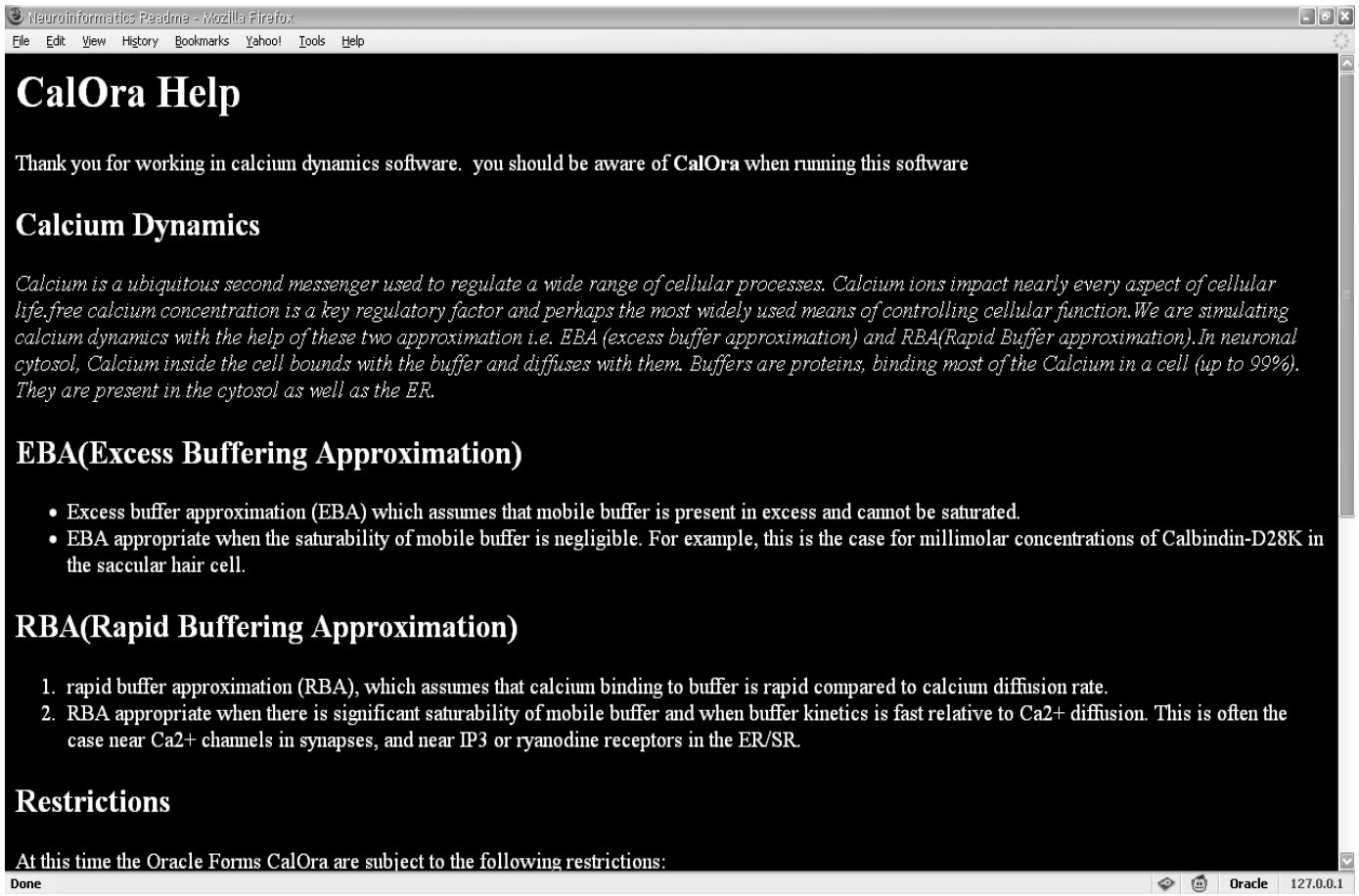

Figure 2. Webpage showing Help section of CalOra where user can aware of its different part 


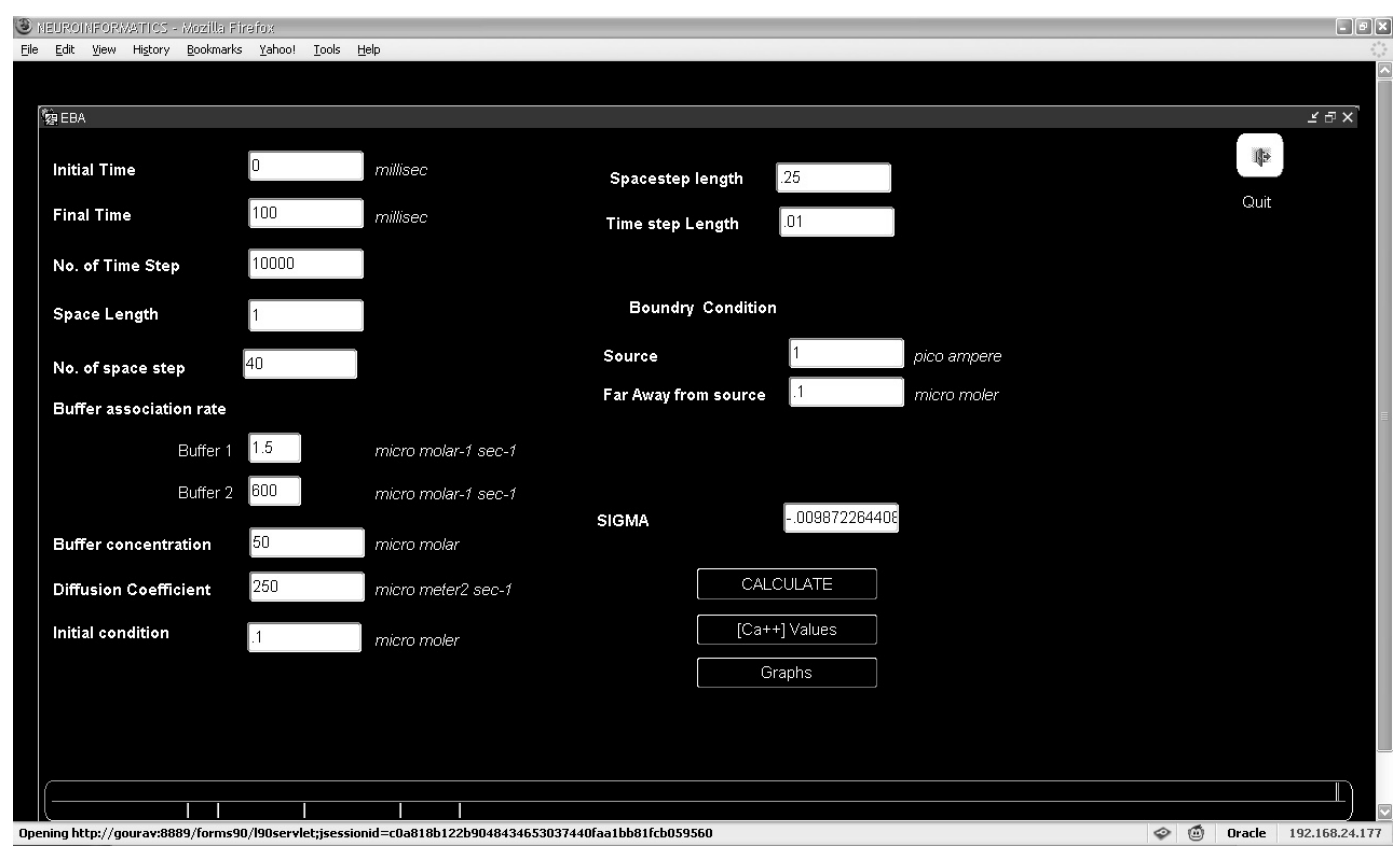

Figure 3. Webpage showing EBA module of CalOra where user can enter different parametric values

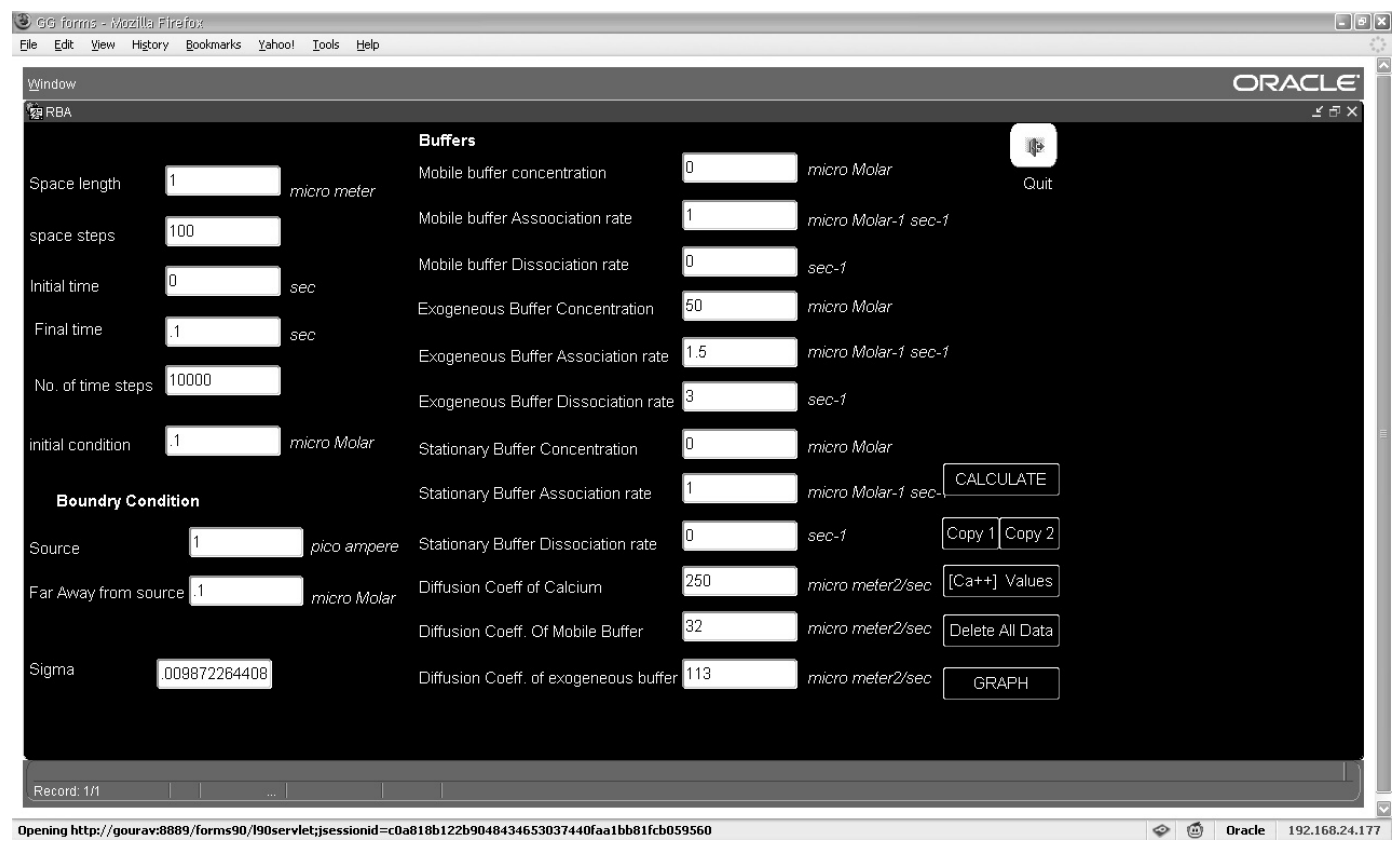

Figure 4. Webpage showing EBA module of CalOra where user can enter different parametric values 


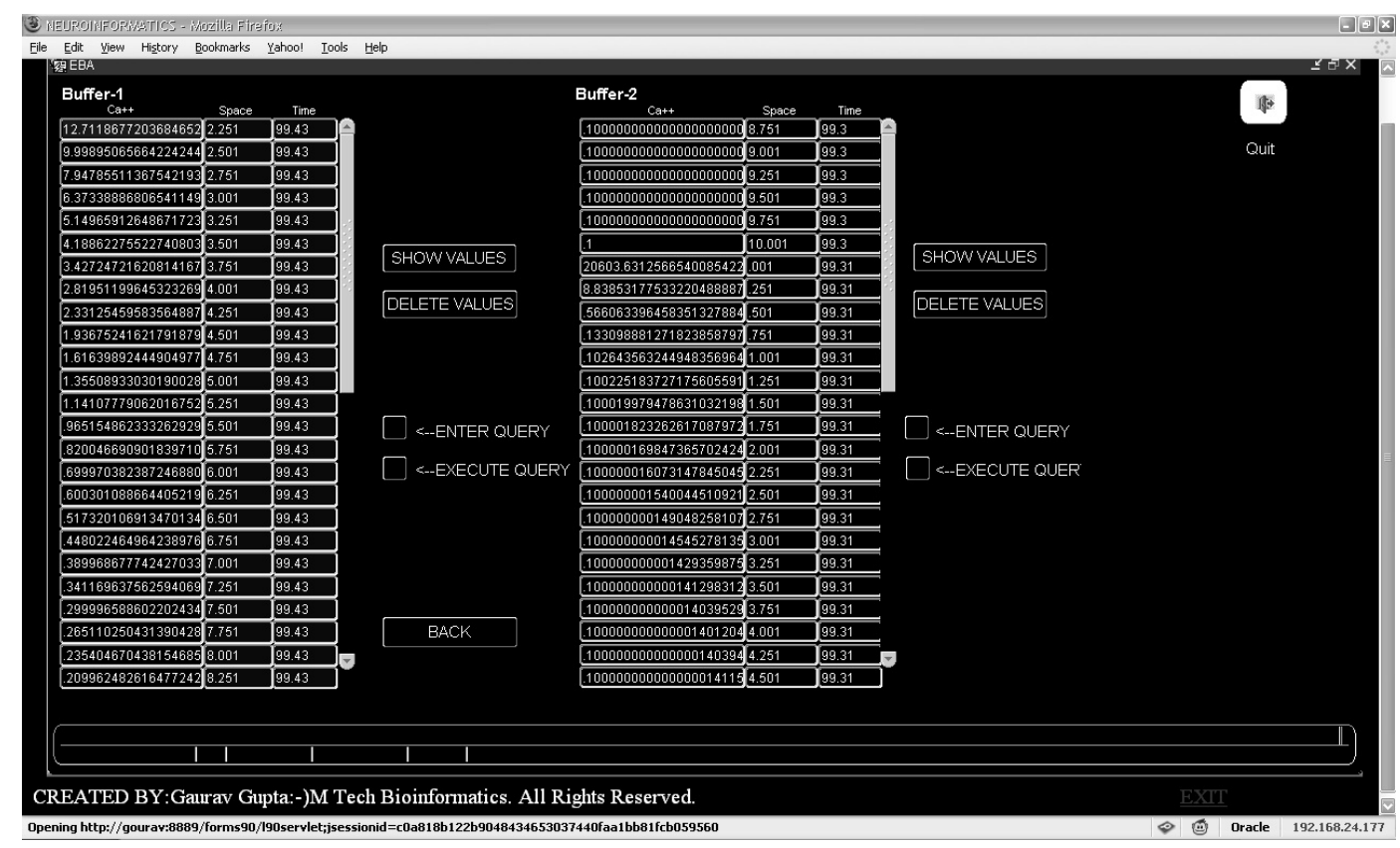

Figure 5. Webpage showing the $\mathrm{Ca}^{2+}$ values of buffer1 (EGTA) and buffer2 (BAPTA) after calculation has done with the parametric values shown in fig 3 of EBA

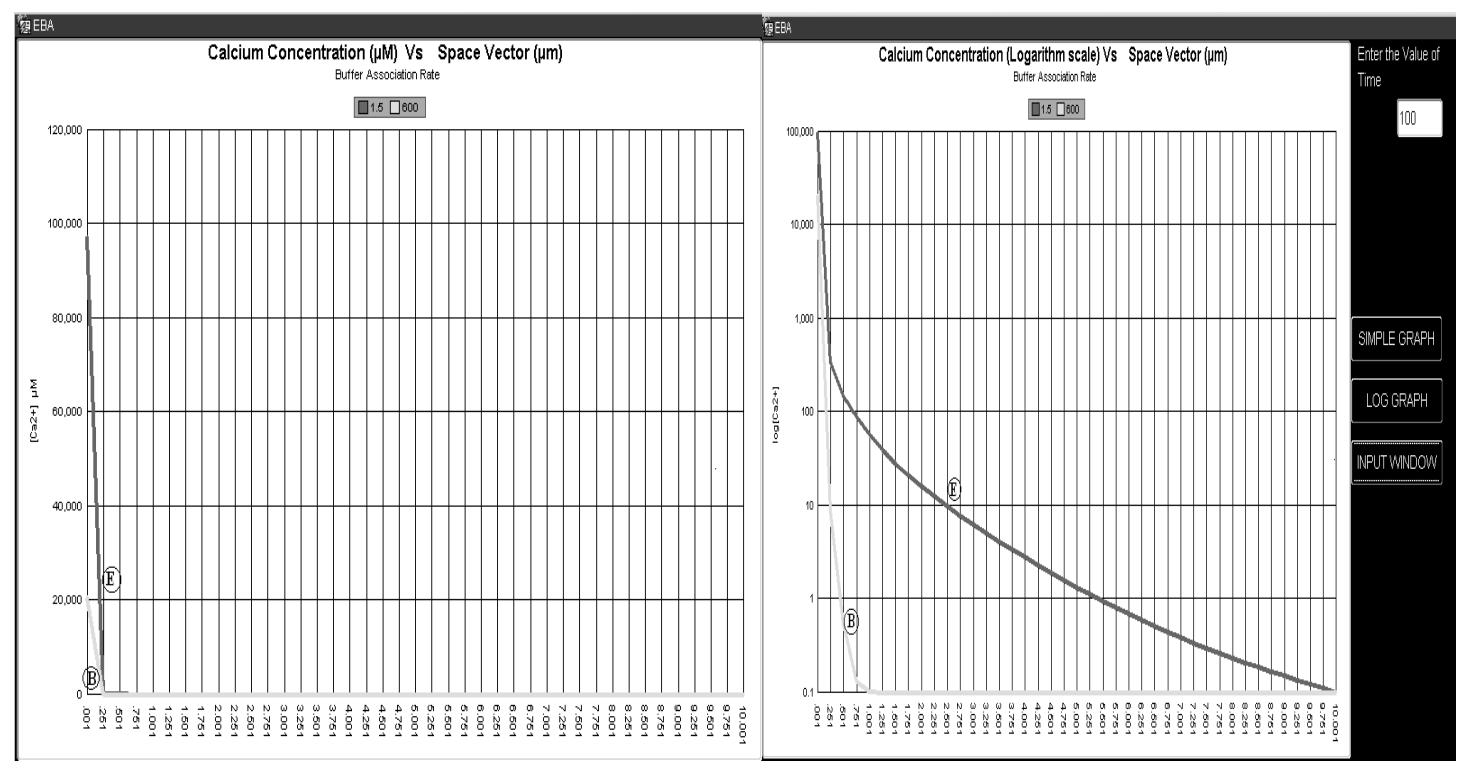

Figure 6. Cropped images showing the results of EBA in the form of simple bar graph and logarithmic graph where (E) \& (B) represent EGTA and BAPTA Buffers respectively 

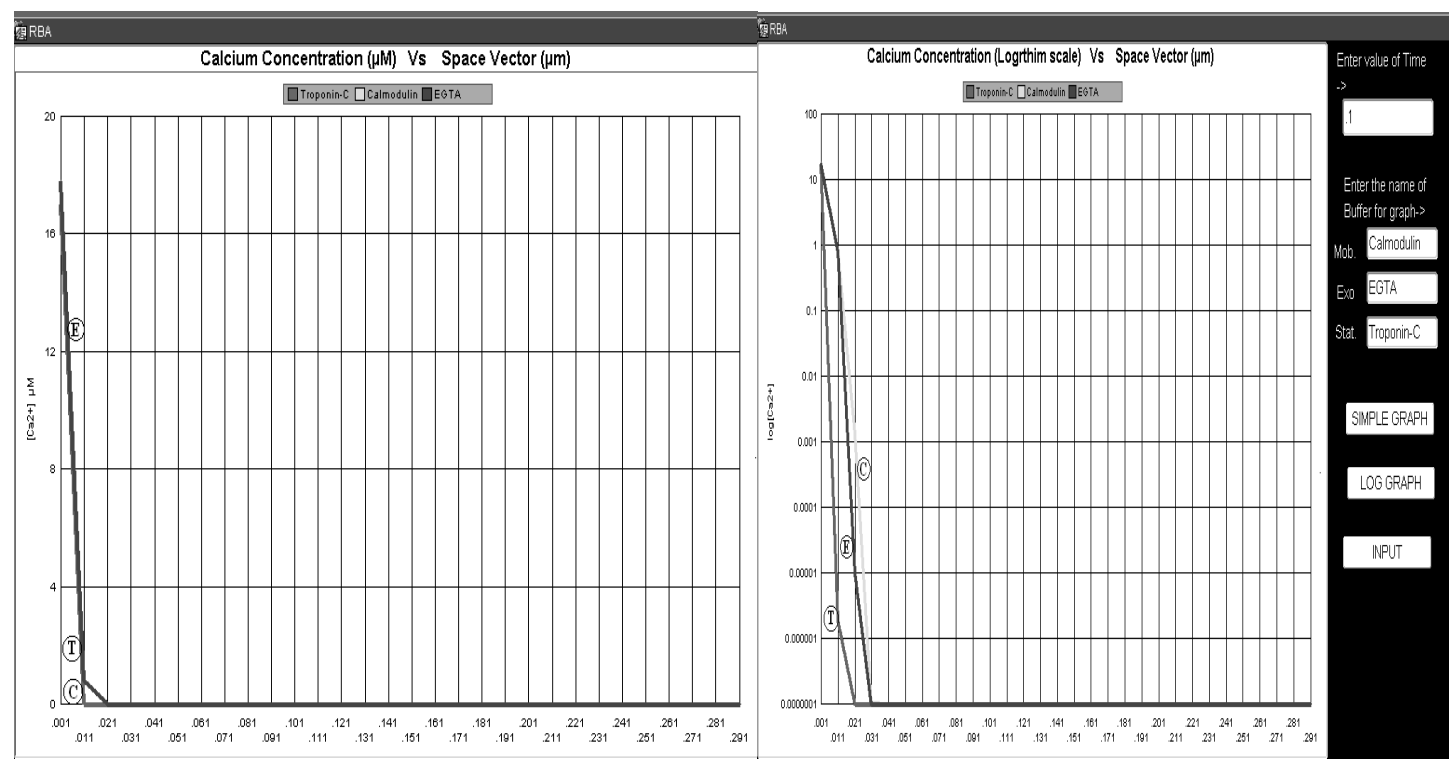

Figure 7. Cropped images showing the results of RBA in the form of simple bar graph and logarithmic graph where (T), (C) and (E) representing the Troponin-C, Calmodulin and EGTA Buffers respectively 